\title{
Art in hospitals: promoting a new humanism
}

\author{
Mariapia Bonanate
}

Can art restore the soul of medicine that the increasingly fierce pursuit of technology has taken away from it, undermining the vital human relationship between doctor and patient? Can painting, literature, philosophy, theatre and dance be turned into new instruments for "creating health"? This is the hope and also the challenge of a vast movement that is spreading across the continents and seeing physicians, healthcare professionals and artists working alongside patients in the attempt to rise to this crucial and exciting challenge.

"When art becomes a pharmakon, it is entitled to be recognised as such, confirming the meaning that the Greeks gave to this word. To today's cultural impoverishment and the economic and political crisis of modern society, which tends to regard art as an added value and not as an expression of need, art is responding with artists capable of producing 'aesthetics of need', thereby confirming a new condition of art, which allows it to have a new social impact. The next step is to produce a new generation of artists able to produce not only aesthetics but also 'ethics of need', in other words artists capable of entering into a Socratic relationship with patients and with disease, with treatment and with those who treat, in a setting of work in progress where there are no hierarchies, only full participation of all those involved" explains eminent art critic Bonito Oliva.

The most important hospitals in the United States, Britain, Canada and France have departments that involve artists in treatments and that, in order to improve understanding of disease, conduct research into the ways in which art affects the mind. Artists in the United States have signed an appeal to Congress to be recognised as a vital and integral and part of the health system. They complain of the difficulties they encounter in getting their work regulated in federal health programmes, even though studies have shown that use of the arts for therapeutic, educational and expressive purposes is extremely useful and a constantly expanding phenomenon. They have shown that where there is use of the arts, quality of life is improved. There is less depression and less anxiety, both in patients and in professionals, but also reduced spending: less drug consumption and related costs, and shorter hospital stays - a remarkable achievement in these times of global economic crisis.

Amedeo Bianco, President of the Italian Federation of Surgeons and Dentists, underlines the importance of this growing phenomenon: "The discoveries in the neurosciences are leading the way. Art, which, in its various and complex manifestations, has accompanied mankind from his most ancient origins, continues to be a deep need of the human spirit. This need becomes more acute in the extreme situations of life, but also in the presence of more elementary emotions, such as the doubt and fear that often accompany birth, illness and death. Art can be a powerful tool for improving treatment, but it is also the driving force of a change in our way of being physicians, patients, health professionals and citizens. If, nowadays, we seem to be witnessing a crisis of identities, this is perhaps an indication that the time has come for a true return to the humanist roots of medicine; to humanism based on respect, listening, a critical spirit, hope and solidarity".

In 2009, HUM MED, the "EuroMediterranean network for the humanisation of medicine" was founded in Turin, Italy. This network serves to connect hospitals, universities, health protection agencies, researchers, anthropologists, architects, economists, and philosophers from Italy, France, the UK, Belgium, Holland, Switzerland, and now Israel too. The network aims to help medicine return to its primary - humanistic and holistic - vocation, and to reintroduce the arts into medical science, a setting that is becoming increasingly high tech and bureaucratic. Art has a great capacity for suggesting ways in which people can interact" explains HUM MED president Dr. Rossana Beccarelli. And she goes on: "Artists today, in the wake of the experiences of the twentiethcentury avant-garde movements, are coming out of their own world and into contact with people, producing art that helps to increase understanding of how we treat and how treatment can be improved". In some of Turin's hospitals (and also in hospitals in other Italian cities), painting, music, dance and theatre are being organised, either by single professionals or as collective community initiatives. Art feeds creativity in medicine and healthcare, becoming a source of inner enrichment, knowledge and insight. It is often able to generate the strength and imagination necessary to accept the life change that illness brings. If a patient is enabled to take charge of his new situation, instead of being left in the dark to endure it passively, then his chances of recovery, or at least of reducing his suffering, increase remarkably. Medicine today has to consider many vital issues that closely affect people's lives, such as water, air and the environment in which they live. It is no longer enough simply to confront the diagnosis".

People have become wary and mistrustful of medicine and the institutions where it is practised, a trend partly driven by the culture of a society that sees illness as an inconvenience and only has time for those who enjoy power, money success and wellbeing (physical and material) - in short, for winners. Accordingly, a person who 


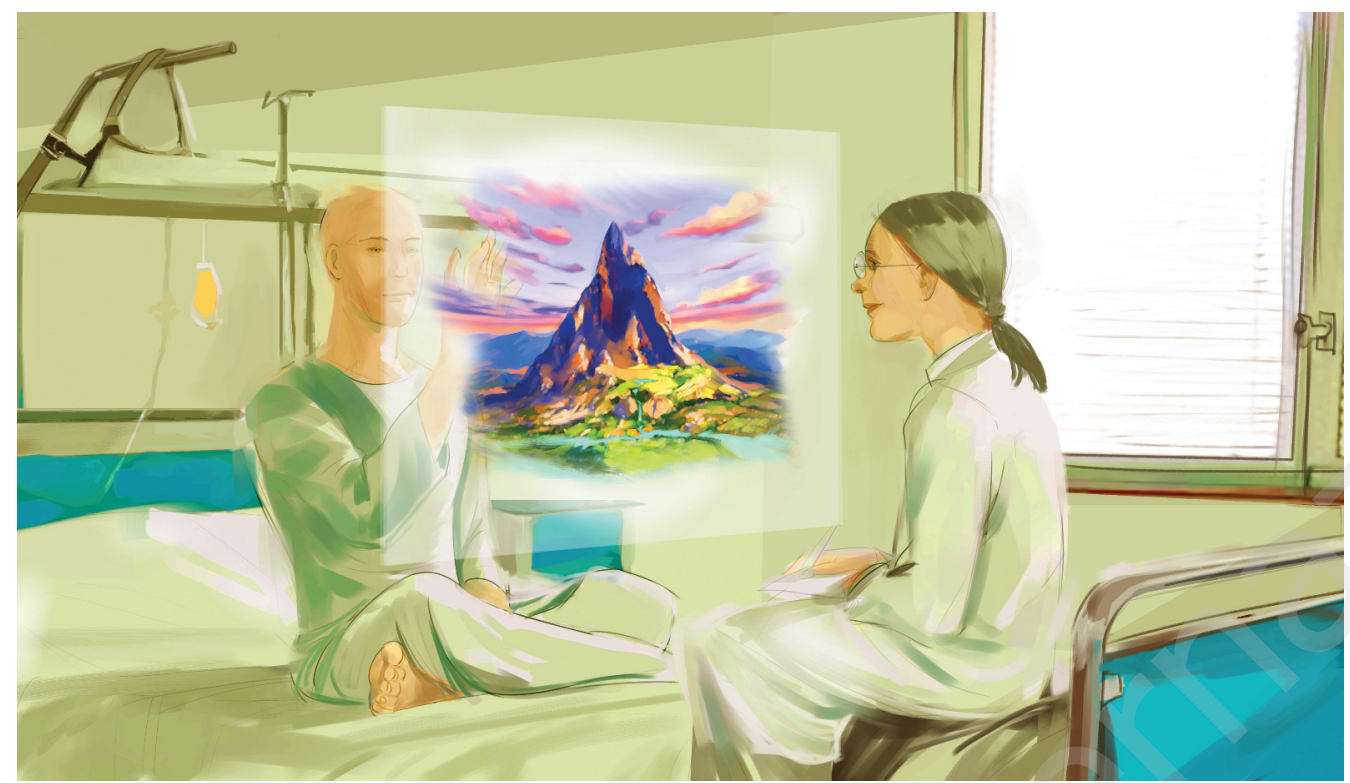

"Digital art" by Marco Ceruti.

is sick becomes a loser, a threat to the circuit of efficiency. No longer a face, a person with a story, a past and a present, all he amounts to is a bed number and a cold, sterile medical record chart. Together with his health, he also loses his identity. In this way, disease, suffering and even death are screened out, while hospitals, in a context of healthcare management increasingly conditioned by financial and political considerations, are allowed to become ghettoes to be forgotten or abandoned. Thus, all the opportunities that disease offers risk being lost, if we continue to focus only on the individual's physical conditions, forgetting his psychological, mental, inner state and the environment in which he lives. When the great journalist Tiziano Terzani developed cancer, he remarked that the real medicine for tackling cancer, "his" cancer, was not increasingly sophisticated drugs or last generation chemotherapy regimens, but rather a corner of his mind in which to re-establish a harmonious relationship both with himself and with the world.

Art can contribute to clinical practice by helping healthcare professionals and patients to accept disease as a moment in one's existence in which it is possible to reinvent one's life, discovering, within it, values and meanings previously overlooked; in short, by bringing out a richness of spirit, human and intellectual, that draws on the whole of life in order to live a part of it to the full.

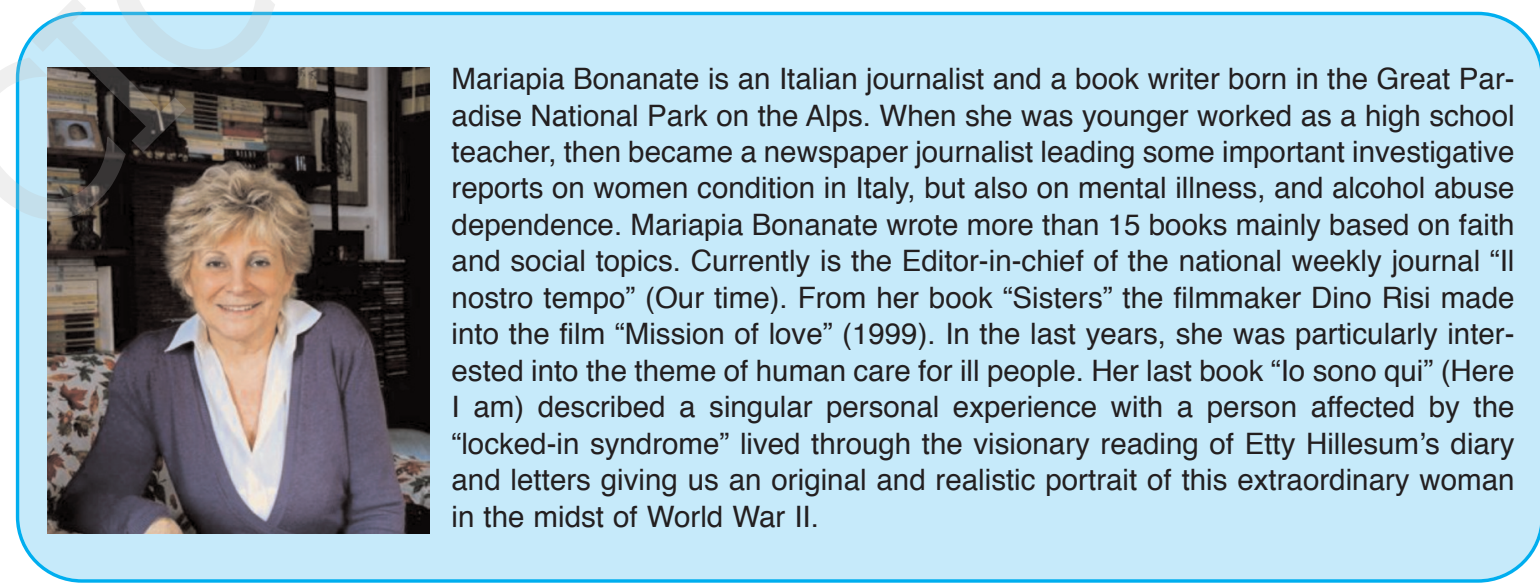

